\title{
Esiste un'alternativa alla matrice dermica acellulare per la gestione delle ferite? Rivisitazione del ruolo di una medicazione bioattiva a base di collagene e acido ialuronico
}

\author{
Matteo Torresetti, Giovanni Di Benedetto, Alessandro Scalise \\ Clinica di Chirurgia Plastica e Ricostruttiva, Università Politecnica delle Marche, Ancona, Italia
}

\begin{abstract}
RIASSUNTO
La guarigione delle ferite è un processo complesso che coinvolge molte interazioni sinergiche tra diverse linee cellulari, citochine, enzimi e fattori di crescita. Negli ultimi anni sono stati introdotti diversi biomateriali e medicazioni bioattive che si ritiene svolgano un ruolo attivo nella guarigione delle ferite. Sia il collagene che l'Acido Ialuronico (AI) sono stati ampiamente adottati per la gestione delle ferite e la sua combinazione, utilizzata come medicazione bioattiva, è stata studiata. Abbiamo riportato la nostra esperienza clinica con una medicazione avanzata composta da AI più collagene eterologo di tipo I applicato su ferite acute e croniche di diversa eziologia in una coorte retrospettiva di 30 pazienti. Tutti i pazienti inclusi avevano ferite cutanee portate a guarigione completa per seconda intenzione, e tutte le ferite sono state trattate utilizzando Bionect Pad ${ }^{\circledR}(\mathrm{BP})$. Se necessario, alcuni casi sono stati gestiti con trattamenti combinati al fine di ottenere un'adeguata preparazione del letto della ferita prima dell'applicazione di BP. Trenta pazienti con età media di 60,2 anni sono stati trattati per ferite di diversa eziologia. La tipologia di ferite trattate includeva ulcere venose, ferite post-traumatiche, complicanze di ferite chirurgiche, lesioni da decubito, ustioni, ulcerazioni peristomali e ulcerazioni cutanee dopo radioterapia. Il tempo medio di guarigione è stato di 31 giorni (range: 21-76 giorni). Sulla base dei nostri risultati incoraggianti, riteniamo che tali medicazioni bioattive possano essere considerate un'alternativa utile e affidabile ad altri metodi di trattamento ben noti e consolidati, come le matrici dermiche acellulari o altre medicazioni avanzate, in casi selezionati.
\end{abstract}

Corrispondenza: Matteo Torresetti, Clinica di Chirurgia Plastica e Ricostruttiva, Università Politecnica delle Marche, Via Conca 71, 60126, Ancona, Italia.

Tel.: +390715963454 .

Fax: +390715963453 .

E-mail: torresetti.matteo@gmail.com

Parole chiave: collagene, acido iauronico, wound healing, medicazioni bioattive, matrici dermiche acellulari.

Contributo degli autori: MT e AS: design dello studio, redazione e revisione del manoscritto, analisi e interpretazione dei dati; GDB e AS: supervisione e coordinazione allo studio, approvazione del manoscritto finale.

Confliti di interesse: Il Prof. Alessandro Scalise ha rapporti finanziari con Fidia Farmaceutici S.p.a. in merito alla collaborazione scientifica nella consulenza in ricerca e sviluppo. Gli altri autori dichiarano l'assenza di conflitti di interesse.

Ricevuto per la pubblicazione: 16 novembre 2020.

Accettato per la pubblicazione: 30 novembre 2020.

This work is licensed under a Creative Commons Attribution NonCommercial 4.0 License (CC BY-NC 4.0).

${ }^{\circ}$ Copyright: the Author(s), 2020

Licensee PAGEPress, Italy

Italian Journal of Wound Care 2020; 4(2):37-44

doi:10.4081/ijwc.2020.70

\section{ABSTRACT}

Wound healing is a complex process, which involves a lot of synergistic interactions between several cell lines, cytokines, enzymes, and growth factors. In the last few years several biomaterials and bioactive dressings, which are considered to play an active role in wound healing have been introduced. Both collagen and Hyaluronic Acid (HA) have been largely adopted for wound management and its combined used as bioactive dressing have been studied. We reported our clinical experience with an advanced wound dressing consisting of HA plus heterologous collagen type I applied on acute and chronic wounds of different aetiology in in a retrospective cohort of 30 patients. All patients included have cutaneous open wounds completely healed by only second intention, and all wounds were treated by using Bionect Pad ${ }(B P)$. If necessary, some cases were managed with combined treatments in order to obtain an adequate wound bed preparation before the application of BP. Thirty patients with an average age of 60,2 years were treated for wounds of different aetiology. Wound types included venous ulcers, posttraumatic wounds, surgical wound complications, pressure sores, burns, peristomal ulcerations and skin ulcerations after radiation therapy The average healing time was 31 days (range: 21-76 days). Basing on our encouraging results, we believe that such bioactive dressings may be considered as a useful and reliable alternative to other well-known and established treatment methods such as acellular dermal matrices or advanced wound dressings in selected cases. 


\section{INTRODUZIONE}

L'Acido Ialuronico (AI), è un glicosaminoglicano presente praticamente in tutti i tessuti umani, dove rappresenta un costituente fondamentale della Matrice Extracellulare (MEC). Da un punto di vista funzionale, mentre in passato l'AI era semplicemente considerato come un composto in grado di riempire gli spazi e assorbire gli urti, è ora evidente che, a seconda del suo peso molecolare e attraverso le interazioni con i suoi recettori primari, le funzioni biologiche dell'AI sono molto più complesse. Il suo importante ruolo biologico nel controllo dell'omeostasi cellulare dell'acqua, grazie al suo alto grado di idratazione, è stato ben stabilito. Poiché le matrici altamente idratate facilitano la migrazione e la proliferazione cellulare, l'AI può supervisionare e regolare il comportamento cellulare e l'interazione cellula-cellula, specialmente nel caso di guarigione delle ferite. Queste funzioni regolatrici e le interazioni con altre macromolecole, dipendono dal peso molecolare e dalla concentrazione di AI. Grazie alle sue peculiari proprietà strutturali, versatilità, alla sua presenza ubiquitaria nei sistemi dei mammiferi, e alla sua biocompatibilità, l'AI rappresenta un'alternativa sicura e affidabile in diverse applicazioni biomediche, come la visco-integrazione, la somministrazione di farmaci, la chirurgia oculare, l'ingegneria dei tessuti e la medicina rigenerativa. Mentre per alcune applicazioni si utilizza l'AI nella sua forma nativa, la funzionalizzazione chimica della macromolecola viene spesso eseguita per adattare il suo tempo di permanenza in vivo e le sue proprietà generali, superando così le implicazioni biologiche della degradazione dell'AI. ${ }^{1-4}$

Il collagene è l'unica molecola proteica a tripla elica, che costituisce la parte principale della matrice dermica extracellulare e rappresenta il $70-80 \%$ del peso secco del derma. Prodotto principalmente dai fibroblasti, il collagene di tipo I comprende circa il $70 \%$ del collagene nella pelle, con il 10\% di tipo III, e funge da impalcatura nel tessuto connettivo. ${ }^{5}$ Il collagene è coinvolto in tutte e 3 le fasi della cascata di guarigione della ferita, stimolando la migrazione cellulare e contribuendo allo sviluppo di nuovi tessuti. Nella ferita cronica, la deposizione di collagene de novo è ritardata o impedita da numerosi fattori, come gli elevati livelli di enzimi coinvolti nella degradazione proteolitica del collagene. Considerate pertanto le proprietà biologiche e strutturali del collagene, il suo utilizzo nel campo della gestione delle ferite è davvero interessante. ${ }^{6}$ Esistono diversi tipi di medicazioni al collagene, che impiegano una varietà di veicoli/agenti combinati come gel, paste, polimeri, Cellulosa Rigenerata Ossidata (CRO) e acido Etilen-Diammina-Tetraacetico (EDTA). Il collagene contenuto in questi prodotti è solitamente derivato da fonti bovine, suine, equine o aviarie, che viene quindi purificato per renderlo non antigenico. La struttura dell'impalcatura della medicazione per ferite funge da guida per il movimento di fibroblasti, macrofagi e cellule epiteliali e promuove la loro migrazione nell'area della ferita. $^{7-8}$ Negli ultimi due decenni, sono stati sviluppati sul mercato diversi prodotti rigenerativi contenenti acido ialuronico e collagene. Questi prodotti rappresentano medicazioni bioriassorbibili per la guarigione delle ferite, e scaffold compatibili con le cellule per l'ingegneria dei tessuti, al fine di migliorare l'efficacia della medicina rigenerativa. ${ }^{9}$ Nonostante il collagene e l'AI vengano ampiamente applicati su medicazioni e scaffold, è necessario considerare alcuni effetti collaterali. Ad esempio, ialuronidasi e collagenasi possono degradare lo scaffold producendo prodotti di scarto, che possono indurre infiammazione e influenzare il processo di guarigione della ferita. L'elevato tasso di degradazione e le scarse proprietà meccaniche del collagene e dell'AI spesso non soddisfano i requisiti dell'applicazione specifica di alcune medicazioni. Per queste ragioni sono stati recentemente fatti molti tentativi di "imitare" la matrice extracellulare naturale (MEC) e diversi scaffold come collagene Condroitin Solfato (CS), collagene - AI e gelatina - CS - AI sono stati prodotti. Nonostante questi scaffold abbiano dimostrato tassi di degradazione inferiori, e una maggiore resistenza meccanica rispetto al solo collagene, la loro biocompatibilità non è risultata del tutto soddisfacente. ${ }^{10}$ Sulla base di queste osservazioni, abbiamo studiato gli effetti di una nuova medicazione bioattiva composta da $\mathrm{AI}$ più collagene eterologo di tipo I [Bionect Pad $\mathbb{R}$ (BP), Fidia Farmaceutici S.p.A., Abano Terme - PD, Italia] applicata su ferite acute e croniche di diversa eziologia. Lo scopo dello studio era di valutare la sicurezza e l'efficacia di questo nuovo prodotto nella gestione delle ferite, rivisitando il loro ruolo come alternativa alle matrici dermiche acellulari in casi selezionati e difficili da trattare. Per quanto di nostra conoscenza, questo è il primo studio sull'applicazione di BP in tutta la letteratura medica.

\section{MATERIALI E METODI}

Tutti i pazienti inclusi nel presente studio avevano ferite cutanee portate a guarigione completa solo per seconda intenzione, in un periodo compreso fra Aprile 2018 e Aprile 2019. Tutte le ferite sono state trattate utilizzando la medicazione BP dalla data di ricovero fino alla data di riepitelizzazione completa. Il protocollo di trattamento prevedeva il cambio della medicazione ogni 4 giorni e una visita ambulatoriale di follow-up ogni 10 giorni al fine di identificare tempestivamente eventuali effetti collaterali o ritardi nella guarigione della ferita. Se necessario, alcuni casi sono stati gestiti con trattamenti combinati al fine di ottenere un'adeguata preparazione del letto della ferita prima dell'applicazione del BP. I pazienti che sono stati trattati solo temporaneamente con BP prima della successiva ricostruzione chirurgica sono stati esclusi. Sono stati registrati i dati demografici, dati riguardanti l'eziologia della ferita, il tipo 
di trattamento combinato e il tempo di guarigione.

\section{RISULTATI}

Negli ultimi 12 mesi abbiamo curato un totale di 30 pazienti con ferite di diversa eziologia utilizzando matrici di BP. L'età media dei pazienti era di 60,2 anni (range: 23-82 anni). Le ferite erano principalmente causate da ulcere venose, seguite da traumi, complicanze della ferita chirurgica, piaghe da decubito, ustioni, ulcerazioni peristomali e ulcerazioni cutanee dopo radioterapia. Nella maggior parte dei pazienti, sono stati necessari trattamenti precedenti combinati tra cui la terapia a pressione negativa, lo sbrigliamento chirurgico o altre medicazioni avanzate. Il tempo medio di guarigione è stato di 31 giorni (range: 21-76 giorni). Non sono stati segnalati effetti collaterali che hanno portato alla sospensione del trattamento. Le principali caratteristiche dei pazienti trattati sono riassunte nella Tabella 1.

\section{CASO N. 2}

Un uomo di 69 anni è stato ricoverato nel nostro ospedale per una ferita post-traumatica del dorso del piede destro con esposizione tendinea. Dopo un primo debridement chirurgico, il paziente è stato sottoposto a trattamento conservativo con terapia a pressione negativa per 3 settimane al fine di ottenere tessuto di granulazione e copertura parziale del tendine. Quindi è stato gestito in modo conservativo con medicazioni a base di BP per 60 giorni e si è ottenuta la guarigione completa della ferita (Figura 1).

\section{CASO N. 3}

Un uomo di 43 anni ha riportato una rottura postraumatica del tendine di Achille. Si è sottoposto a ricostruzione chirurgica del tendine lesionato, ma e il decorso postoperatorio è stato complicato da una deiscenza della ferita con esposizione tendinea che è stata gestita in modo conservativo mediante terapia a pressione negativa per 6 settimane. Successivamente, una volta che il letto della ferita è stato adeguatamente coperto dal tessuto di granulazione, è stato utilizzato $\mathrm{BP}$ e si è ottenuta una guarigione completa della ferita dopo 76 giorni (Figura 2).

\section{CASO N. 6}

Un uomo di 36 anni, paraplegico, con numerose lesioni da decubito ricorrenti a livello dell'ischio e della regione sacrale, è stato visitato in regime ambulatoriale per

Tabella 1. Caratteristiche principali dei pazienti trattati.

\begin{tabular}{|c|c|c|c|}
\hline Dati demografici & Eziologia della ferita & Trattamenti combinati & Tempo di guarigione \\
\hline \multirow[t]{8}{*}{$\begin{array}{l}\text { Età media: } 60,2 \text { anni } \\
\text { (range: } 23-82 \text { anni) }\end{array}$} & Ulcere venose (tot. 9) & $\begin{array}{l}\text { Terapia a pressione negativa } \\
\text { (tot. 4) }\end{array}$ & $\begin{array}{l}\text { Tempo medio: } 31 \text { giorni } \\
\text { (range: } 21-76 \text { giorni) }\end{array}$ \\
\hline & Ferite postraumatiche (tot. 6) & $\begin{array}{l}\text { Altre medicazioni avanzate } \\
\text { (tot. 15) }\end{array}$ & \\
\hline & $\begin{array}{l}\text { Complicanze di ferrite chirurgiche } \\
\text { (tot. 4) }\end{array}$ & Nessuno (tot. 11) & \\
\hline & Lesioni da pressione (tot. 4) & & \\
\hline & Ustioni (tot. 4) & & \\
\hline & Ulcere peristomali (tot. 2) & & \\
\hline & Radioterapia (tot. 1) & & \\
\hline & Tot. 30 pazienti & Tot. 30 pazienti & \\
\hline
\end{tabular}

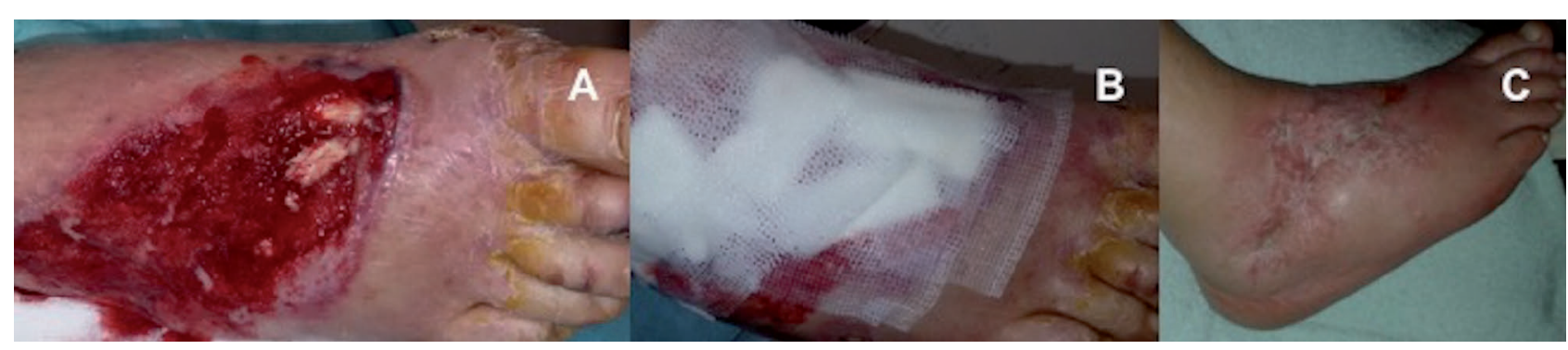

Figura 1. Ferita postraumatica del piede destro con esposizione tendinea dopo sbrigliamento chirurgico e terapia a pressione negativa (A); Applicazione di Bionect Pad sul letto della ferita (B), visita di follow-up a 45 giorni (C). 
un'ulteriore recidiva della lesione da pressione sacrale. In precedenza era stato sottoposto a diverse ricostruzioni chirurgiche delle lesioni da decubito utilizzando lembi locali, con conseguenti cicatrici chirurgiche multiple che hanno precluso una nuova ricostruzione con lembi. Pertanto abbiamo optato per una gestione conservativa con medicazioni avanzate della ferita a base di collagenasi e idrofibra contenente argento, che sono state continuate fino ad ottenere un'adeguata preparazione del letto della ferita. Dopo 4 settimane di trattamento, è stata applicata una matrice di BP e la guarigione completa dell'ulcera si è verificata dopo 6 settimane (Figura 3).

\section{CASO N. 8}

Un uomo di 68 anni è stato sottoposto ad intervento chirurgico per un carcinoma a cellule di Merkel (MCC) della regione posteriore della gamba. È stato sottoposto a rimozione chirurgica di MCC e ricostruzione con matrice dermica acellulare, e successivo innesto cutaneo a spessore parziale. Veniva quindi sottoposto a radioterapia adiuvante e, dopo circa 1 mese, ha sviluppato ulcerazioni cutanee che sono state gestite in modo conservativo utilizzando BP. Una guarigione completa della ferita è stata raggiunta 3 settimane dopo (Figura 4, A-D).

\section{DISCUSSIONE}

La guarigione delle ferite è un processo complesso, che coinvolge molte interazioni sinergiche tra diverse linee cellulari, citochine, enzimi e fattori di crescita. La ricerca sulla guarigione delle ferite include l'identificazione di quegli elementi che accelerano il processo di guarigione, materiali che funzionano come efficaci sostituti cutanei e segnali responsabili di innescare la guarigione. Le medicazioni bioattive sono considerate quelle che svolgono un ruolo attivo nella guarigione delle ferite. Esempi di materiali bioattivi che fanno parte di queste medicazioni sono collagene, chitosano, acido ialuronico e pectina. ${ }^{11}$ Non è realistico aspettarsi che una singola medicazione possa avere tutte le caratteristiche in grado di soddisfare tutte le esigenze generali per la guarigione delle ferite. Pertanto, l'obiettivo principale dovrebbe essere la creazione di una medicazione versatile che abbia la capacità di influenzare positivamente la maggior parte dei tipi di ferite. Ciò può essere ottenuto solo attraverso un approccio multidimensionale che utilizza additivi bioattivi per un effetto mirato. ${ }^{12}$ L'AI è stato ampiamente utilizzato come medicazione per ferite e diversi biomateriali e bioscaffold a base di AI sono prodotti in diverse forme tra cui idrogel, tubi, lamine e reti. I biomateriali e i bioscaffold di AI hanno molti vantaggi, in particolare la loro natura non allergogena e non infiammatoria a differenza di altri materiali (ad esempio i derivati del chitosano). Tuttavia, la resistenza in vivo degli impianti a base di AI e dell'AI iniettato, dipende dalla loro capacità di resistere

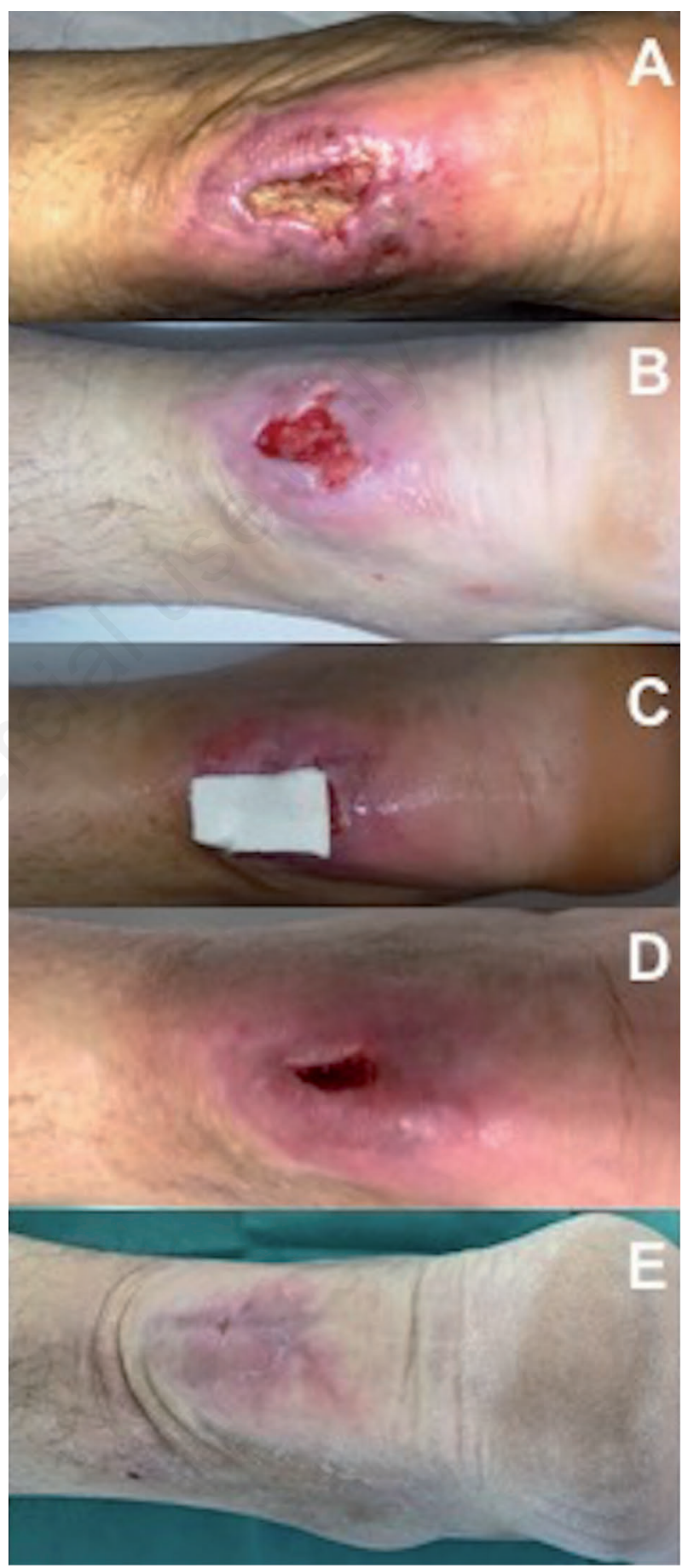

Figura 2. Deiscenza della ferita con esposizione del tendine di Achille (A); dopo un' adeguata preparazione del letto della ferita è stata applicata una matrice di Bionect Pad (B-C). Visita di follow-up dopo 50 giorni (D); la guarigione completa della ferita è stata ottenuta dopo 76 giorni (E). 
alla degradazione da parte delle ialuronidasi e delle specie reattive dell'ossigeno e dell'azoto (ROS/RNS), limitando così il loro utilizzo in modo efficiente. Per superare i problemi con la degradazione dell'AI, sono state sfruttate le proprietà fisico-chimiche dell'AI, inclusa la disponibilità di gruppi funzionali reattivi sull'AI, facilitando così le modificazioni chimiche sull'AI e rendendolo un materiale biocompatibile per l'utilizzo nella rigenerazione dei tessuti. ${ }^{1-4}$

Numerosi fattori che influenzano la guarigione delle ferite possono comprometterne l'intero processo. Nella ferita cronica, la deposizione di collagene de novo è ritardata; inoltre, il reclutamento dei fibroblasti è anch'esso ritardato e l'espressione del gene del collagene nei fibroblasti è soppressa. Anche i fattori ambientali influenzano il livello di collagene nel letto della ferita cronica. Tra questi fattori vi sono 2 classi di enzimi, i cui livelli sono noti per essere elevati nelle ferite croniche: le Metalloproteinasi della Matrice (MMP) e l'elastasi. Le MMP sono implicate nella degradazione proteolitica del collagene intatto nativo e dei frammenti di collagene parzialmente degradati. L'elevato rapporto tra MMP e inibitori tissutali di MMP porta a un'eccessiva degradazione della matrice extracellulare. L'elastasi è anche coinvolta nel processo di guarigione grazie al suo ruolo nel convertire i pro-MMP (il precursore naturale degli MMP) in MMP attivi. L'attività dell'elastasi è elevata nella ferita cronica, contribuendo così fortemente al carico di MMP nella ferita stessa. In sintesi, la ferita cronica è caratterizzata sia da una diminuzione della deposizione di collagene che da un aumento della degradazione del collagene. ${ }^{6}$ Pertanto l'uso di medicazioni al collagene nella gestione della ferita può sembrare attraente grazie all'inibizione o disattivazione delle MMP, all'aumentata produzione e permeazione dei fibroblasti, all'aiuto nell'assorbimento e biodisponibilità della fibronectina, per la conservazione di leucociti, macrofagi, fibroblasti e cellule epiteliali e supporto nel mantenimento del microambiente chimico e termostatico della ferita. ${ }^{5}$ Le medicazioni al collagene caratterizzate da un $\mathrm{pH}$ basso, possono abbassare il $\mathrm{pH}$ del liquido della ferita,
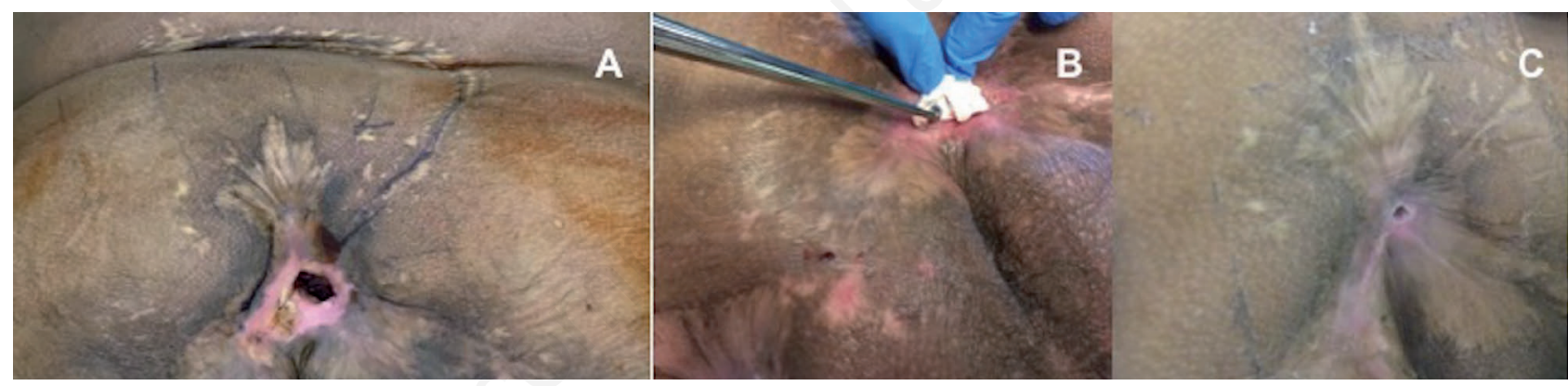

Figura 3. Recidiva di una lesione da pressione sacrale in un paziente paraplegico (A); completa guarigione della ferita dopo 6 settimane di trattamento con Bionect Pad (B-C).

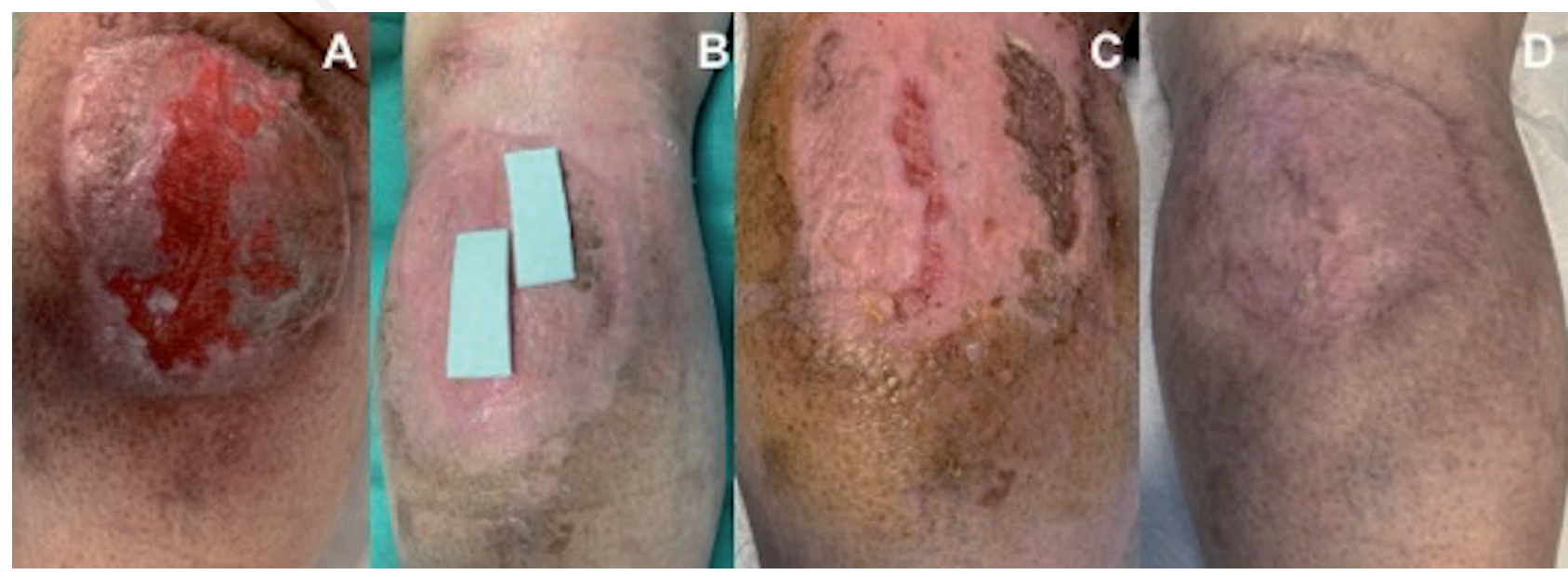

Figura 4. Ulcerazioni cutanee dovute alla radioterapia della regione posteriore della gamba (A); visite di follow-up dopo l'applicazione di Bionect Pad a 15 giorni (B-C) e 21 giorni (D). 
diminuendo così il rischio di colonizzazione batterica secondaria. Il collagene ha siti di legame per i fibroblasti e ha un effetto chemiotattico su queste cellule. La dimensione dei pori della schiuma di collagene è importante per la migrazione cellulare e per la formazione di capillari e del tessuto cicatriziale. ${ }^{7,8}$

Il biomateriale ideale dovrebbe pertanto avere diverse caratteristiche per promuovere la riparazione o la rigenerazione dei tessuti all'interno delle ferite croniche: i) attrarre di cellule in grado di sintetizzare nuovo tessuto nel sito della ferita; ii) promuovere la proliferazione cellulare; ii) fornire uno scaffold non immunogenico e riassorbibile per la migrazione cellulare e la deposizione di matrice; iv) organizzare la deposizione della nuova MEC; v) modulare l'attività proteolitica; vi) assorbire e neutralizzare i radicali liberi e/o ioni metallici in eccesso. È chiaro che i composti di collagene soddisfano molti di questi criteri. ${ }^{13}$ Diverse forme di collagene possono essere utilizzate nella pratica clinica come spugne, iniettabili, pellicole e membrane, medicazioni e innesti cutanei. Le spugne di collagene sono particolarmente utili nella guarigione delle ferite perché le spugne impiantate sono infiltrate da tessuto connettivo amorfo contenente Glicosaminoglicani (GAG), fibronectina e nuovo collagene, seguito da varie cellule (principalmente fibroblasti e macrofagi). A seconda del grado di reticolazione, la spugna di collagene viene degradata dalle collagenasi in frammenti peptidici e amminoacidi in 3-6 settimane e l'impianto viene quindi sostituito dal collagene nativo di tipo I prodotto dai fibroblasti. ${ }^{14}$

Dal 2003, Kuroyanagi et al. (citato in Huang et al.) ${ }^{9}$ ha condotto una serie di esperimenti su una matrice spugnosa a doppio strato costituita da uno strato di AI, e un secondo strato di collagene e suoi peptidi derivati. Questi due strati di spugna legati in modo covalente per formare un Sostituto Dermico di Coltura (SDC) sono stati quindi applicati come medicazione per ferite, ed è stato osservato nel modello animale che la CDS autologa può fornire una guarigione efficiente. Due anni dopo gli stessi autori hanno rivelato in pazienti umani che i fibroblasti nella CDS hanno prodotto diversi fattori bioattivi, come fattori di crescita cellulare e componenti della matrice extracellulare che sono importanti per la guarigione della ferita, migliorando così le condizioni della ferita, consentendo al sito della ferita di adattarsi ad un successivo trapianto autologo di pelle e riducendo i tempi medi di guarigione di ferite cutanee a tutto spessore. ${ }^{9}$

Gli scaffold compositi hanno solitamente due o più biomateriali combinati per migliorare le loro proprietà meccaniche, o la loro efficienza funzionale o entrambi. Il rapporto di miscelazione e il grado di reticolazione dei polimeri selezionati sono davvero cruciali per determinare la biostabilità, la resistenza meccanica e la microstruttura degli scaffold compositi. Alcuni studi riportano che gli scaffold composti da collagene, AI e CS hanno effetti benefici se usati per la rigenerazione della pelle, delle ossa o della cartilagine. Nel 2012 Kondo et al. hanno studiato gli effetti di una medicazione composta da acido ialuronico (AI) e spugna di collagene $(\mathrm{Col})$ contenente Fattore di Crescita Epidermico (FCE) su vari parametri di guarigione della ferita in vitro e in vivo, e hanno osservato che la combinazione di AI, Col ed FCE promuove la guarigione delle ferite stimolando la funzione dei fibroblasti. ${ }^{15}$ Recentemente Drobnik et al. ha studiato se un tri-copolimero di collagene con l'aggiunta di $\mathrm{AI}$ e $\mathrm{CS}(\mathrm{Col}+\mathrm{AI}+$ $\mathrm{CS})$ può migliorare le colture di cellule nervose embrionali e fibroblasti della ferita, determinando così l'utilità di questo tri-copolimero per l'ingegneria dei tessuti. Questo studio ha scoperto che le cellule seminate in scaffold di tri-copolimeri hanno dimostrato una migliore attività metabolica rispetto a quelle in scaffold composti da solo collagene. Si credeva che questa reazione fosse dovuta all'acido ialuronico che modifica la struttura del collagene, consentendo la formazione di interconnessioni tra $\mathrm{i}$ pori, che supporta la migrazione delle cellule ai pori interni, consente una migliore comunicazione tra le cellule e migliora la nutrizione delle cellule seminate. ${ }^{16}$

Kirk et al. hanno studiato nel 2013 la caratterizzazione meccanica e biocompatibile di una medicazione a base di collagene-acido ialuronico reticolato. Basandosi sull'osservazione che i materiali non reticolati sono molto più sensibili alla degradazione della proteasi rispetto al materiale reticolato con EDC (1-ethyl-3-(3- dimethyl aminopropyl)carbodiimide) carbodiimide, hanno rivelato che la stabilizzazione dei compositi ricostituiti di collagene-AI, utilizzando legami incrociati chimici e/o fisici, ha portato alla realizzazione di biomateriali con buona biocompatibilità, maggior potenziale di differenziazione cellulare, maggiore stabilità, senza effetti citotossici o genotossici nel modello animale. Inoltre hanno anche dimostrato che il processo per generare la spugna di collagene-AI reticolato con EDC ha portato alla deposizione omogenea dell'AI attraverso le fibre di collagene senza effetti negativi sulla struttura del collagene stesso o sulla dimensione dei pori, che sono fondamentali per il legame cellulare, la migrazione e proliferazione. Inoltre, il processo di reticolazione non ha influenzato negativamente la forza biomeccanica dello scaffold in collagene. ${ }^{17}$

Mathews et al. nel 2014 hanno studiato uno scaffold con un tripolimero biomimetico, costituito da chitosano, collagene di tipo 1 e acido ialuronico, che ha supportato la proliferazione e la differenziazione delle cellule staminali mesenchimali umane derivate dal midollo osseo per l'ingegneria del tessuto osseo. ${ }^{18}$

Matsiko et al. nel 2012 hanno rivelato che l'incorporazione di AI in uno scaffold a base di collagene ha provocato una stimolazione significativa dell'infiltrazione di cellule staminali mesenchimali nello scaffold; inoltre, 
questi scaffold hanno mostrato una significativa accelerazione dell'espressione genica in fase iniziale del collagene di tipo II e della produzione di matrice cartilaginea. ${ }^{19}$

Alcuni autori hanno anche proposto di ottenere nuovi scaffold compositi, con morfologia e proprietà chimicofisiche adeguate per sistemi di somministrazione controllata di farmaci. Vulpe et al. nel 2018 hanno studiato uno scaffold reticolato a base di collagene, AI e sericina, che ha dimostrato di avere una struttura porosa, una forza e una stabilità adeguate per i sistemi di somministrazione controllata di farmaci con potenziale utilizzo nell'ingegneria dei tessuti cutanei. ${ }^{20}$

Nonostante gli studi incentrati sull'uso singolo di collagene o AI siano stati ampiamente riportati in tutta la letteratura, le indagini cliniche strettamente mirate alle medicazioni con composti bioattivi sono però ancora marginali.

I biomateriali trovano largo impiego nel campo della chirurgia ricostruttiva e della guarigione delle ferite, per le loro peculiari caratteristiche di sicurezza, affidabilità e facilità di utilizzo. La nostra lunga esperienza clinica con le matrici dermiche acellulari a base di collagene ${ }^{21-24}$ pone alcune importanti considerazioni sulle loro differenze con le medicazioni bioattive per ferite. Innanzitutto, le ADM a base di collagene sono davvero utili per la rigenerazione del neoderma e fungono da copertura temporanea della ferita prima dell'innesto cutaneo; tuttavia, di solito richiedono almeno due passaggi chirurgici e il ricovero in ospedale. Al contrario, le medicazioni per ferite a base di collagene potrebbero essere facilmente applicate e gestite in regime ambulatoriale, e potrebbero non richiedere una ricostruzione chirurgica con innesti cutanei. In secondo luogo, le medicazioni bioattive per ferite con collagene e AI sfruttano sia il potenziale del collagene nell'assemblare lo scaffold per l'adesione e la crescita dei fibroblasti, sia il potenziale dell'AI nel controllo dell'idratazione dei tessuti, garantendo così un letto umido della ferita che è fondamentale per un corretto processo riparativo. La combinazione di questi due componenti porta ad un effetto sinergico con un completo ripristino della matrice extracellulare; inoltre, producono un composto stabile che può esercitare un effetto durevole sul letto della ferita.

Inoltre, le ADM sono solitamente dispositivi costosi e potrebbero non essere disponibili in alcune strutture; al contrario, le medicazioni bioattive possono potenzialmente far risparmiare sui costi a causa del loro minor numero di visite ambulatoriali e di cambio della medicazione, e di una gestione della ferita extraospedaliera. Tuttavia, la nostra esperienza clinica suggerisce che le ADM rimangono ancora il cardine del trattamento in quei casi con difetti tissutali a tutto spessore e con esposizione ossea, come alternativa alla chirurgia ricostruttiva con lembi. Infatti, le medicazioni bioattive per ferite sono state utilizzate nel nostro studio solo in quelle lesioni con un fondo di ferita ben vascolarizzato, e il loro potenziale di copertura ossea non è stato ancora studiato.

Sono tuttavia presenti alcune limitazioni nel nostro studio. Esso infatti è uno studio di coorte retrospettivo che ha un bias di selezione intrinseco. Inoltre, non è stato eseguito alcun confronto con un gruppo di controllo con standard di cura, come ADM o altre medicazioni avanzate per le ferite. Saranno pertanto necessari ulteriori studi per esaminare i potenziali benefici clinici di questa opzione di trattamento in grandi coorti di pazienti con un gruppo di controllo con standard di cure.

\section{CONCLUSIONI}

I risultati del presente studio mostrano che le medicazioni composite potrebbero essere utili sia per lesioni acute che croniche di diversa eziologia; a volte può essere necessario un uso combinato con altre medicazioni avanzate o altri dispositivi medici come la terapia a pressione negativa, per ottenere un fondo lesionale ben vascolarizzato. La buona biocompatibilità, la maggiore stabilità, duttilità e le proprietà biologiche complessive di questi composti bioattivi, rendono tali medicazioni un'alternativa affidabile e utile ad altre medicazioni avanzate o matrici acellulari per la medicina rigenerativa e la guarigione delle ferite. Tuttavia, saranno necessari ulteriori studi per definire il ruolo appropriato di queste medicazioni bioattive nel campo della guarigione delle ferite.

\section{BIBLIOGRAFIA}

1. Reitinger S, Lepperdinger G. Hyaluronan, a ready choice to fuel regeneration: a mini-review. Gerontology 2013;59:716. doi: 10.1159/000342200.

2. Hemshekhar M, Thushara RM, Chandranayaka S, et al. Emerging roles of hyaluronic acid bioscaffolds in tissue engineering and regenerative medicine. Int J Biol Macromol 2016;86:917-28. doi: 10.1016/j.ijbiomac.2016.02.032.

3. Serban MA, Skardal A. Hyaluronan chemistries for threedimensional matrix applications. Matrix Biol 2019;7879:337-345. doi: 10.1016/j.matbio.2018.02.010.

4. Zhao N, Wang X, Qin L, et al. Effect of hyaluronic acid in bone formation and its applications in dentistry. J Biomed Mater Res A 2016;104:1560-9. doi: 10.1002/jbm.a.35681.

5. Rangaraj A, Harding K, Leaper D. Role of collagen in wound management. Wounds UK, 2011;7.

6. Fleck CA, Simman R. Modern collagen wound dressings: function and purpose. J Am Col Certif Wound Spec 2011;2:50-4. doi: 10.1016/j.jcws.2010.12.003.

7. Brett D. A review of collagen and collagen-based wound dressings. Wounds 2008;20:347-56.

8. Pallaske F, Pallaske A, Herklotz K, Boese-Landgraf J. The significance of collagen dressings in wound management: a review. J Wound Care 2018;27:692-702. doi: 10.12968/ jowc.2018.27.10.692. 
9. Huang LLH, Chen YA, Zhuo ZY, et al. Medical Applications of Collagen and Hyaluronan in Regenerative Medicine. Adv Exp Med Biol 2018;1077:285-306. doi: 10.1007/978-98113-0947-2_15.

10. Wang W, Zhang M, Lu W, et al. Cross-linked collagen-chondroitin sulfate-hyaluronic acid imitating extracellular matrix as scaffold for dermal tissue engineering. Tissue Eng Part C Methods 2010;16:269-79. doi: 10.1089/ten.TEC.2009.0161.

11. Mir M, Ali MN, Barakullah A, et al. Synthetic polymeric biomaterials for wound healing: a review. Prog Biomater 2018;7:1-21. doi: 10.1007/s40204-018-0083-4.

12. Mandla S, Davenport Huyer L, Radisic M. Review: Multimodal bioactive material approaches for wound healing. APL Bioeng 2018;2:021503. doi: 10.1063/1.5026773.

13. Gould LJ. Topical collagen-based biomaterials for chronic wounds: rationale and clinical application. Adv Wound Care (New Rochelle) 2016;5:19-31. doi: 10.1089/wound. 2014. 0595.

14. Chattopadhyay S, Raines RT. Review collagen-based biomaterials for wound healing. Biopolymers 2014;101:82133. doi: 10.1002/bip. 22486.

15. Kondo S, Kuroyanagi Y. Development of a wound dressing composed of hyaluronic acid and collagen sponge with epidermal growth factor. J Biomater Sci Polym Ed 2012;23:629-43. doi: 10.1163/092050611X555687.

16. Drobnik J, Pietrucha K, Piera L, et al. Collagenous scaffolds supplemented with hyaluronic acid and chondroitin sulfate used for wound fibroblast and embryonic nerve cell culture. Adv Clin Exp Med 2017;26:223-230. doi: 10.17219/ acem/ 62835.

17. Kirk JF, Ritter G, Finger I, et al. Mechanical and biocompatible characterization of a cross-linked collagen- hyaluronic acid wound dressing. Biomatter 2013;3. doi: 10.4161/biom. 25633 .

18. Mathews S, Bhonde R, Gupta PK, Totey S. Novel biomimetic tripolymer scaffolds consisting of chitosan, collagen type 1, and hyaluronic acid for bone marrow-derived human mesenchymal stem cells-based bone tissue engineering. J Biomed Mater Res B Appl Biomater 2014;102:182534. doi: 10.1002/jbm.b.33152.

19. Matsiko A, Levingstone TJ, O'Brien FJ, Gleeson JP. Addition of hyaluronic acid improves cellular infiltration and promotes early-stage chondrogenesis in a collagen-based scaffold for cartilage tissue engineering. J Mech Behav Biomed Mater 2012;11:41-52. doi: 10.1016/j.jmbbm.2011. 11.012.

20. Vulpe R, Popa M, Picton L, et al. Scaffolds based on collagen, hyaluronan and sericin with potential applications as controlled drug delivery system. J Nanosci Nanotechnol 2018;18:1528-1533. doi: 10.1166/jnn.2018.15182.

21. Grassetti L, Torresetti M, Scalise A, et al. Acellular dermal matrices and paraffinoma: a modern tool for a nearly obsolete disease. Arch Plast Surg 2017;44:234-237. doi: 10.5999/aps.2017.44.3.234.

22. Scalise A, Torresetti M, Verdini F, et al. Acellular dermal matrix and heel reconstruction: a new prospective. J Appl Biomater Funct Mater 2017;15:e376-e381. doi: 10.5301/ jabfm.5000357.

23. Torresetti M, Scalise A, Di Benedetto G. Acellular dermal matrix for rhinophyma: Is it worth it? A new case report and review of literature. Int J Surg Case Rep 2019;59:120-123. doi: 10.1016/j.ijscr.2019.05.013.

24. Scalise A, Torresetti M, Di Benedetto G. Reconstruction of full-thickness soft tissue defects with integra: risk factors and treatment algorithm. Plast Reconstr Surg Glob Open 2020;8:e3099. doi: 10.1097/GOX.0000000000003099 